\title{
Experimental Investigation of the Painlevé Paradox in a Robotic System
}

\author{
Zhen Zhao, Caishan Liu, Wei Ma, Bin Chen \\ State Key Laboratory for Turbulence and Complex Systems, College of Engineering \\ Peking University, China, 100871
}

\begin{abstract}
This paper aims at experimentally investigating the dynamical behaviors when a system of rigid bodies undergoes so-called paradoxical situations. An experimental setup corresponding to the analytical model presented in our prior work [28] is developed, in which a two-link robotic system comes into contact with a moving rail. The experimental results clearly show that a tangential impact does exist at the contact point and takes a peculiar property well coinciding with the maximum dissipation principle stated in [11] by Moreau; the relative tangential velocity of the contact point must immediately approach zero once a Painlevé paradox occurs. After the tangential impact, a bouncing motion may be excited and is influenced by the speed of the moving rail. We adopt the tangential impact rule presented in [28] to determine the post-impact velocities of the system, and use an event-driven algorithm to perform numerical simulations. The qualitative comparisons between the numerical and experimental results are carried out and show good agreements. This study not only presents an experimental support for the shock assumption related to the problem of the Painlevé paradox, but can also find its applications in better understanding the instability phenomena appearing in robotic systems.
\end{abstract}

Keyworsds: Painlevé paradox; robotic system; instability phenomenon; impulsive dynamics;

\section{Introduction}

It is well-known that the rigid body model for mechanical systems with unilateral constraints and friction may possess some singularities at which the dynamical equations will have multiple solutions or even no solution at all. The classical Painlevé example, where a planar slender rod slides on a rough surface, represents the simplest system with such singularities. Recently the interest in understanding the physical phenomena corresponding to the singular situations has witnessed a substantial increase [1-30]. Rich information and a good overview on the subject can be found in the excellent book written by Brogliato [5], which contains a wide variety of the problem-of-interest and a long list of references.

*Email: lcs@mech.pku.edu.cn 
When Coulomb friction is coupled to unilateral constraints, rigid body models may have no solution for certain configurations. An important viewpoint adopted by many authors is that a shock should then exist at the contact point. Since the shock occurs in a special situation without normal velocity and friction is considered to be the main cause for its occurrence, different nomenclatures can be found in the literature, such as the impact without collision (IW/OC), frictional catastrophe, or tangential impact [11, 12 , 16, 19]. According to the shock assumption, some crucial results can be deduced and the problem of the Painlevé paradox seems to be solvable. For example, recent development related to the time-stepping numerical method indicates that the singularity of rigid body model can be successfully avoided if the contact forces is allowed to be impulsive [24-25,30-31], thus confirming a fact observed numerically in [11]. Obviously the shock assumption is fundamental for the problem of Painlevé paradox, and the validation from experiments should be introduced. One aim of this paper is to develop an experimental method to support the shock assumption.

The Painlevé phenomenon was firstly discovered in the classical Painlevé's example (a slender rod that slides on a rough plane), and many excellent theoretical results are motivated from the simple system. However, using the example to serve as the experimental model creates a lot of difficulties. For instance, the coefficient of friction in the system has to be greater than $4 / 3$ for the occurrence of the Painlevé paradox, a large value rarely found in practical materials [16]. Meanwhile, the sliding of a rod under gravity is also difficult to be implemented in practice. Therefore, it is crucial to search for a new example that can involve the paradox and can be easily implemented by experiments. Such examples have been presented in [15, 28], where it has been shown that the Painlevé paradoxes may occur for arbitrarily small values of the coefficient of friction.

Recent studies have indicated that the Painlevé paradox may be a common phenomenon and could be found in a variety of different applications such as robotic manipulation, legged locomotion, and vehicle braking systems [15, 26, 27, 28]. Especially, according to our recent work for a robotic system that comes into contact with a moving belt [28], the Painlevé paradox will appear even though the coefficient of friction takes a very small value. In this paper, we will use the analytical model presented in [28] to develop an experimental setup for the demonstration of the dynamical behaviors associated with the Painlevé paradox.

According to the theoretical analysis shown in [28], the Painlevé paradox can be found by setting the robotic system within a paradoxical configuration, which depends on the practical value of the coefficient of friction. By conducting the shock assumption into the paradoxical situation of the robotic system, and using the Darboux-Keller's method $[21,32-37]$, it is also shown that the tangential impact will take a peculiar property; the relative motion of the contact point must be immediately brought into a stick in tangential direction. Therefore, the shock assumption can be verified by observing whether there is a tangential stick in paradoxical situations. In our experiments, two laser-vibrameters with precise accuracy will be used to carefully measure the changes of the contact relative velocities of the robotic system.

Using numerical methods to reproduce the dynamical behaviors is significant for the analysis of mechanical systems. It is obvious that a jump rule related to the tangential impact should be provided for systems involving paradoxical situations. Since the tangential impact will excite a bouncing motion that can make the system contain differ- 
ent modes of motion such as slipping phases, collisions with friction, and flying without contact, an event-driven algorithm will be used to perform the numerical simulations. Qualitative comparisons between the numerical and experimental results are carried out.

Based on the numerical analysis and the experimental observation, two instability phenomena exhibited in robotic systems are discovered: one that is the bouncing motion induced by a tangential impact, the other that is related to a slip-stick motion that appears in the robotic system without the occurrence of the Painlevé paradox. The first case indicates that the problem of the Painlevé paradox will extremely influence the controllability of the robotic system, as pointed out by Brogliato [4]. The second case shows that the stick-slip motion is periodic when the rail moves with a constant velocity.

The organization of the study is as follows. Section 2 presents the description of the model for a two-link manipulator. The key theoretical results developed in [28] for the paradoxical situation will be reviewed in section 3. The experimental setup and the phenomena associated with the paradoxical situations will be exhibited in section 4 . Section 5 provides the comparison between the numerical and experimental results. We conclude in section 6 with a summary and the potential application of our study.

\section{A two-link manipulator with unilateral constraint}

This section will first conduct the model of a two-link robotic system, and then find the condition for the occurrence of the Painlevé paradox by using an LCP (Linear Complementarity Problem) method, a theory for non-smooth dynamics established by Moreau $[38,39]$ and then extended into the multibody systems by Pfeiffer and Glocker [2].

The manipulator is shown in figure.1, which consists of two identical rods with length

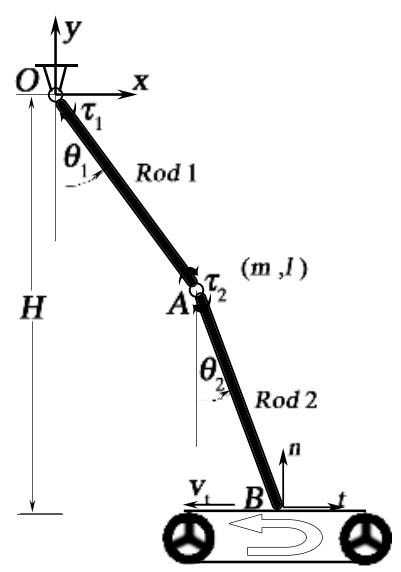

Figure 1: Two-link manipulator contacting with a constantly moving belt

$l$ and mass $m$ and comes into contact with a moving belt with velocity $v_{t}$. The external torques $\tau_{1}$ and $\tau_{2}$ are applied on the joints $O$ and $A$. $H$ is the height from the fixed point $O$ to the rough surface. The joint angles $\theta_{1}$ and $\theta_{2}$ are selected as the generalized coordinates of the system when unconstrained by contact, and their positive values are assigned along the counter-clockwise direction.

We set an inertial coordinate frame $O x y$ attached at the joint $O$, and suppose that a 
local inertial coordinate frame $(B, t, n)$ with origin located at contact point $B$, is defined such that $n$ is normal to the contact surface and $(t, n)$ forms a right-handed coordinate system. $\left(F_{t}, F_{n}\right)$ represent the contact forces in the tangential and normal direction. The components in $O x y$ for the contact point $B$ can be expressed by the generalized coordinates.

$$
\mathbf{x}=\left[\begin{array}{c}
x_{t} \\
x_{n}
\end{array}\right]=\left[\begin{array}{c}
l\left(\sin \theta_{1}+\sin \theta_{2}\right) \\
-l\left(\cos \theta_{1}+\cos \theta_{2}\right)
\end{array}\right]
$$

These kinematics can yield the contact Jacobian matrix $\mathbf{K}$ that relates velocities and accelerations of the contact point $B$ to the generalized coordinates through the relations

$$
\begin{aligned}
& \dot{\mathbf{x}}=\mathbf{K}^{\mathbf{T}} \dot{\mathbf{q}} \\
& \ddot{\mathbf{x}}=\mathbf{K}^{\mathbf{T}} \ddot{\mathbf{q}}+\mathbf{S}
\end{aligned}
$$

Where

$$
\mathbf{K}=\left[\begin{array}{c}
K_{1} \\
K_{2}
\end{array}\right]=\left[\begin{array}{ll}
l \cos \theta_{1} & l \sin \theta_{1} \\
l \cos \theta_{2} & l \sin \theta_{2}
\end{array}\right], \quad \mathbf{S}=\left[\begin{array}{c}
S_{1} \\
S_{2}
\end{array}\right]=\left[\begin{array}{c}
-l\left(\dot{\theta}_{1}^{2} \sin \theta_{1}+\dot{\theta}_{2}^{2} \sin \theta_{2}\right) \\
l\left(\dot{\theta}_{1}^{2} \cos \theta_{1}+\dot{\theta}_{2}^{2} \cos \theta_{2}\right)
\end{array}\right]
$$

The governing equations for the system with persistent contact can be written as

$$
\ddot{\mathbf{q}}=\mathbf{M}^{-1} \mathbf{K F}+\mathbf{M}^{-1}(-\mathbf{R}+\mathbf{W})
$$

Where

$$
\begin{gathered}
\mathbf{M}=\left[\begin{array}{cc}
4 m l^{2} / 3 & m l^{2} \cos \left(\theta_{1}-\theta_{2}\right) / 2 \\
m l^{2} \cos \left(\theta_{1}-\theta_{2}\right) / 2 & m l^{2} / 3
\end{array}\right] \quad \mathbf{F}=\left[\begin{array}{c}
F_{t} \\
F_{n}
\end{array}\right] \quad \ddot{\mathbf{q}}=\left[\begin{array}{c}
\ddot{\theta}_{1} \\
\ddot{\theta_{2}}
\end{array}\right] \\
\mathbf{W}=\left[\begin{array}{c}
\tau_{1}-\tau_{2}-3 m g l \sin \theta_{1} / 2 \\
\tau_{2}-m g l \sin \theta_{2} / 2
\end{array}\right] \quad \mathbf{R}=\left[\begin{array}{c}
m l^{2} \dot{\theta}_{2}^{2} \sin \left(\theta_{1}-\theta_{2}\right) / 2 \\
m l^{2} \dot{\theta}_{1}^{2} \sin \left(\theta_{1}-\theta_{2}\right) / 2
\end{array}\right]
\end{gathered}
$$

The substitution of Equation (3) into Equation (4) leads to

$$
\ddot{\mathbf{x}}=\mathbf{Q F}+\mathbf{K}^{\mathbf{T}} \mathbf{M}^{-1}(-\mathbf{R}+\mathbf{W})+\mathbf{S}
$$

where

$$
\mathbf{Q}=\mathbf{K}^{\mathbf{T}} \mathbf{M}^{-\mathbf{1}} \mathbf{K}=\left[\begin{array}{ll}
Q_{11} & Q_{12} \\
Q_{21} & Q_{22}
\end{array}\right]
$$

is a matrix that depends only on the configuration of the system.

Fact 1. $\mathbf{Q}$ is a symmetric and positive definite matrix, since $\mathbf{M}$ is a symmetric positive matrix and $\mathbf{K}$ is full-rank in most cases ( $i E ; \mathbf{K}$ may have singularities only in some extreme configurations).

The relative velocity between the contact point and the belt is

$$
\dot{x}_{r}=x_{t}-v_{t}=l\left(\dot{\theta}_{1} \cos \theta_{1}+\dot{\theta}_{2} \cos \theta_{2}\right)-v_{t}
$$

If $\dot{x}_{r} \neq 0$, the manipulator will slip on the moving belt, otherwise the tip sticks on the belt. Defining a velocity-dependent coefficient of friction $\mu$, in which $\mu=\mu_{0}$ for $\dot{x}_{r}<0$ 
and $\mu=-\mu_{0}$ for $\dot{x}_{r}>0$, the relationship between the tangential and the normal contact force by the Coulomb's frictional law can be expressed as

$$
F_{t}=\mu \cdot F_{n}
$$

During the slip mode, the dynamical equations in tangential and normal directions take the following form.

$$
\begin{aligned}
& \ddot{x}_{n}=A(\mathbf{q}, \mu) \cdot F_{n}+B(\mathbf{q}, \dot{\mathbf{q}}) \\
& \ddot{x}_{t}=C(\mathbf{q}, \mu) \cdot F_{n}+D(\mathbf{q}, \dot{\mathbf{q}})
\end{aligned}
$$

where

$$
\begin{array}{ll}
A(\mathbf{q}, \mu)=\mu Q_{21}+Q_{22}, & B(\mathbf{q}, \dot{\mathbf{q}})=\mathbf{K}_{2}^{T} \mathbf{M}^{-1}(-\mathbf{R}+\mathbf{W})+S_{2} \\
C(\mathbf{q}, \mu)=\mu Q_{11}+Q_{12}, & D(\mathbf{q}, \dot{\mathbf{q}})=\mathbf{K}_{1}^{T} \mathbf{M}^{-1}(-\mathbf{R}+\mathbf{W})+S_{1}
\end{array}
$$

Combining the Signorini complementarity condition $\left(\ddot{x}_{n} \geq 0, F_{n} \geq 0\right.$ and $\left.\ddot{x}_{n} \cdot F_{n}=0\right)$ with equation (9) gives the standard formulation of an LCP whose unknown is $F_{n}$ and whose matrices (here a scalar) are $A(\mathbf{q}, \mu)$ and $B(\mathbf{q}, \dot{\mathbf{q}})$. Obviously, negative values of $A$ will make this LCP possess multiple solutions or no solution at all. As illustrated by many authors a so-called impact without collision occurs because of the configurations in which $A<0$ and $B<0$.

More interestingly, by observing the ingredients of the coefficient $A$, we can find that the paradoxical situation just depends on the configuration of the system and the velocitydependent coefficient of friction. Therefore, for a given coefficient of friction, the Painlevé paradox is allowed to occur only when the system takes the paradoxical configurations, for which $A<0$. In other words, we can rely on the practical value of the coefficient of friction to determine the initial configuration that can make the Painlevé paradox appear.

\section{The properties of the tangential impact and the impact rule}

By conducting the shock assumption into the paradoxical situations, the theoretical description related to the properties of the tangential impact and the impact rule for the robotic system have been presented in [28]. In this section some key results will be introduced.

\subsection{The properties of the tangential impact}

In order to consider the coupling between normal and tangential motion, the experience of an impact with friction must be carefully investigated. By assigning to the impact duration a very short but not infinitesimal time, Darboux [37] and Keller [34] developed a method that yields a set of differential equations with respect to the normal impulse, a 'time-like' independent variable. These nonlinear differential equations describe the impulsive behaviors (the impact dynamics), such that the singularities of impacts due to friction can be successfully avoided $[35,36]$. This method has been extended for the 
investigation of the properties of the tangential impact [28].

Let us set the impact duration as $\left[t_{0}, t_{f}\right]$ and divide this short time into much smaller intervals $\left[t_{i}, t_{i+1}\right]$. Integrating equation (5) and ignoring the contribution of the finite forces on $\left[t_{i}, t_{i+1}\right]$, yields the following differential equations of motion:

$$
\left\{\begin{array}{l}
d \dot{x}_{t}=Q_{11} d P_{t}+Q_{12} d P_{n} \\
d \dot{x}_{n}=Q_{21} d P_{t}+Q_{22} d P_{n}
\end{array}\right.
$$

where

$$
d P_{t}=\int_{t_{i}}^{t_{i+1}} F_{t} d t, d P_{n}=\int_{t_{i}}^{t_{i+1}} F_{n} d t
$$

are the changes of tangential and normal impulses on $\left[t_{i}, t_{i+1}\right]$, respectively.

Now applying the relationship $d P_{t} / d P_{n}=\mu$ defined by the Coulomb's frictional law into equation (11) directly leads to

$$
\left\{\begin{array}{l}
d \dot{x_{t}}=\left(\mu Q_{11}+Q_{12}\right) d P_{n} \\
d \dot{x_{n}}=\left(\mu Q_{21}+Q_{22}\right) d P_{n}
\end{array}\right.
$$

where

$$
d \dot{x}_{t}=\int_{t_{i}}^{t_{i+1}} \ddot{x}_{t} d t, d \dot{x}_{n}=\int_{t_{i}}^{t_{i+1}} \ddot{x}_{n} d t
$$

are the changes of the tangential and normal velocities, respectively.

During the interval $\left[t_{i}, t_{i+1}\right]$, the normal impulse is a strictly monotone function of time. This permits to think of it as a 'time-like' independent variable, and to perform a time-scale of the shock dynamics. Thus, equation (12) is a set of first order ordinary differential equations with respect to $d P_{n}$, which varies like a time variable. Combining equation (12) with the condition for the occurrence of the Painlevé paradox, we can deduce the property of the tangential impacts, which is elucidated by the following theorem.

Theorem.1 The impulsive process induced by the Painlevé paradox will first result in a normal compressional process, and then immediately bring the relative tangential velocity of the contact point to zero. After that the tangential motion of the contact point will stick on the contact surface, while the normal motion of the contact point will continue to be compressional until the normal velocity equals zero. Then an expansion phase in the normal direction is carried out to make the impact finish.

Proof:

We will use fact 1 for the properties of matrix $Q$, the condition for the occurrence of Painlevé paradox, and the Coulomb's frictional law to prove the property of the tangential impact.

Matrix $Q$ can be thought of as a constant matrix due to the little change of the configuration in an impulsive process. So the elements in $Q$ satisfy the following relationships based on fact 1:

$$
Q_{22}>0, Q_{11}>0, Q_{11} Q_{22}>Q_{21}^{2}
$$


During the slip mode, the condition for the occurrence of Painlevé paradox permits to write that

$$
A=\mu Q_{21}+Q_{22}<0,-\mu Q_{21}>Q_{22}
$$

In the case $\dot{x}_{t}<0$, we have $\mu=\mu_{0}>0$, so $Q_{21}<0$. Therefore,

$$
-\mu Q_{21} Q_{11}>Q_{22} Q_{11}>Q_{21}^{2}
$$

and

$$
\left(\mu Q_{11}+Q_{12}\right)>0
$$

Similarly, if $\dot{x}_{t}>0$, we can obtain the following inequality

$$
\left(\mu Q_{11}+Q_{12}\right)<0
$$

Thus, these two cases $\left(\dot{x}_{t}>0\right.$ and $\left.\dot{x}_{t}<0\right)$ can be expressed by using a uniform inequality:

$$
\dot{x}_{t}\left(\mu Q_{11}+Q_{12}\right)<0
$$

The above inequality indicates that the magnitude of the tangential velocity always decreases when the sliding friction is sustained. Since the condition $A<0$ cannot be removed if the tangential velocity is not set to zero, the tangential motion of the contact point must reach the point of slip stopping.

Before slip stops, we can find from the second equation of (12) that the contact point will take a normal velocity penetrating into the contact surface, with an increment for its magnitude ( $A<0$ and the initial value equal to zero). Due to the coupling between the normal and tangential impulses, a compressional process in the normal direction is generated once the paradox appears. Before slip stopping, $A<0$ is always satisfied, so that the magnitude of the normal velocity will continue to increase. So the tangential slip at the contact point stops in the compressional process where the normal velocity is not equal to zero.

Once the tangential speed vanishes, the tangential motion might stick on the contact surface or continue to slip, depending on the property of dry friction. Setting $d \dot{x}_{t}=0$ in equation (11), one can obtain the following inequality

$$
\left|d P_{t} / d P_{n}\right|=\left|Q_{12} / Q_{11}\right|<\mu_{0}
$$

Usually the static coefficient of friction, $\mu_{s}$ is larger than the sliding coefficient $\mu_{0}$. The above inequality indicates that contact forces must enter into the interior of the friction cone once the tangential velocity disappears. So stick should occur at the instant when the tangential velocity vanishes. During the tangential impact, no other additional impulses are applied on the system. The stick mode can be preserved until the impulsive process finishes. Therefore, we can conclude that the relative tangential speed after tangential impact must equal zero. This well coincides with the maximum dissipation principle stated in [11] by Moreau.

Once stick occurs, $d P_{n}$ and $d P_{t}$ cannot be connected linearly by the coefficient of friction $\mu$, and must satisfy the relationship defined by the first equation in (11) by setting $d \dot{x}_{t}=0$. This relationship can make the normal velocity of the contact point decrease and reach zero. After that an expansion phase will occur in order to release the energy 
accumulated in the compressional phase. The expansion phase can be governed by an impact law such as the Poisson's or Stronge's laws [33].

We can summarize the process of the tangential impact as follows: the friction will first result in a compressional motion in the normal direction and then bring the relative tangential motion from slip to stick. Then, sticking motion will persist until the contact constraint is released. The normal motion at the contact point will continue to be compressional from the instant of stick appearance to the time when the normal velocity vanishes. After that an expansion phase is carried out to make the impact terminate.

\subsection{The impact rule for the tangential impact}

Assigning a duration $\left[t_{0}, t_{f}\right]$ to the tangential impact, we can split such an impulsive process into three periods. The first one is a sliding compressional period denoted as $\left[t_{0}, t_{1}\right]$, where $t_{1}$ is related to the instant of stick appearing. The second one is a sticking compressional period denoted as $\left[t_{1}, t_{2}\right]$, where $t_{2}$ corresponds to the instant when the normal velocity vanishes. The third one is a sticking restitution period denoted as $\left[t_{2}, t_{f}\right]$, which describes the expansion process of the normal motion, defined by using the Poisson's law for normal impact.

Sliding compressional period, $\left[t_{0}, t_{1}\right]$

Let us set $\dot{x}_{t}^{0}$ as the initial speed of slip at $t_{0}$. At $t=t_{1}$, the tangential velocity $\dot{x}_{t}^{1}$ will be equal to zero. Thus, the change of the normal impulse can be obtained by integrating the first equation of (12)

$$
P_{n}^{1}=-\frac{\dot{x}_{t}^{0}}{\mu Q_{11}+Q_{12}}
$$

By considering the initial value of the normal velocity $\dot{x}_{n}^{0}=0$, we can obtain the normal velocity $\dot{x}_{n}^{1}$ at $t_{1}$ by integrating the second equation in (12) and by using expression (13):

$$
\dot{x}_{n}^{1}=\left(\mu Q_{21}+Q_{22}\right) P_{n}^{1}=-\frac{\mu Q_{11}+Q_{12}}{\mu Q_{21}+Q_{22}} \dot{x}_{t}^{0}
$$

Sticking compressional period, $\left[t_{1}, t_{2}\right]$

The stick at the contact point implies the following relationship:

$$
d \dot{x}_{t}=0, d P_{t} / d P_{n}=-\left(Q_{12} / Q_{11}\right)
$$

Combining (15) with the second equation of (11), one can deduce the differential equation for the normal motion in the stick mode:

$$
d \dot{x}_{n}=\left(-\frac{Q_{12}^{2}}{Q_{11}}+Q_{22}\right) d P_{n}
$$

Due to $\dot{x}_{n}^{2}=0$ at the end of this period, we can easily obtain the change of the normal impulse $P_{n}^{2}$ at $t_{2}$ :

$$
P_{n}^{2}=\frac{Q_{11} \dot{x}_{n}^{1}}{Q_{12}^{2}-Q_{11} Q_{22}}=-\frac{Q_{11}\left(\mu Q_{11}+Q_{12}\right)}{\left(Q_{12}^{2}-Q_{11} Q_{22}\right)\left(\mu Q_{21}+Q_{22}\right)} \dot{x}_{t}^{0}
$$


Sticking restitutional period, $\left[t_{2}, t_{f}\right]$

This period represents an expansion process of the normal motion. The expansion impulse $P_{n}^{r}$ can be obtained by using the Poisson's coefficient $e_{p}$

$$
e_{p}=\frac{P_{n}^{r}}{P_{n}^{c}}
$$

where $P_{n}^{c}=P_{n}^{1}+P_{n}^{2}$ is the compressional impulse in the normal direction. So we have

$$
P_{n}^{r}=e_{p} P_{n}^{c}=e_{p}\left(P_{n}^{1}+P_{n}^{2}\right)
$$

At the beginning of this period, the normal speed $\dot{x}_{n}^{2}=0$. Meanwhile, the normal motion during this period will be governed by equation (16) since stick in tangential direction is preserved. Thus, at the end of this period the normal speed $\dot{x}_{n}^{f}$ can be expressed as

$$
\dot{x}_{n}^{f}=\left(-\frac{Q_{12}^{2}}{Q_{11}}+Q_{22}\right) P_{n}^{r}
$$

Clearly the post-impact velocity in the normal direction, $\dot{x}_{n}^{f}$ is not equal to zero except for $e_{p}=0$ for the tangential impact without any initial normal velocity. In other words, after the tangential impact the contact point will leave the contact surface with a certain velocity in the normal direction. Nevertheless, the tangential velocity at the contact point $\dot{x}_{t}^{f}$ must be equal to zero when the tangential impact finishes.

By integrating equation (4) and neglecting the contribution of the finite forces, the changes of the generalized velocities of the system due to the tangential impact can also be calculated:

$$
\left[\begin{array}{c}
\Delta \dot{\theta}_{1} \\
\Delta \dot{\theta}_{2}
\end{array}\right]=M^{-1} K\left[\begin{array}{c}
P_{t} \\
P_{n}
\end{array}\right]
$$

where $\Delta \dot{\theta}_{1}$ and $\Delta \dot{\theta}_{2}$ represent the changes of the generated velocities, respectively. $P_{t}$ and $P_{n}$ are the total impulses in the tangential and normal directions.

\section{The dynamical behavior related to the paradoxical situ- ation}

In this section, we will present the experimental setup for the robotic system that corresponds to the analytical model described in above section. According to the coefficient of friction estimated from experiments, we will firstly determine the paradoxical configuration that can make the Painlevé paradox appear. By setting the system with the paradoxical configuration and initially establishing a contact constraint, we can observe the dynamical behavior associated with the paradoxical situation. The paradoxical phenomena will be demonstrated by showing the velocities of the contact point. The influence of the rail's speed on the bouncing motion generated due to the tangential impact will be exhibited. In addition, how the dynamical behaviors of the robotic system evolve into a shock from a normal configuration to the singular one will be demonstrated experimentally, and the stick-slip phenomena appearing in the robotic system will be investigated. 


\subsection{The description of experiment}

The experimental setup of the robotic system formed by using two identical aluminium cylindrical bars $(m=0.12 \mathrm{~kg}, l=0.21 \mathrm{~m})$ connected with revolute joints, see figure 2 . The upper revolute joint is used to connect the system with a fixed bracket that contains a slot to make the height of the system adjustable. A semi-spherical head made of plastic material is installed on the contact end in order to make the contact achieve relatively uniform conditions during the motion. The belt used in the analytical model is replaced by a steel rail, which moves along the horizonal direction, dragging a rope by hand.

A passive torque is provided by a torsional spring mounted at the middle revolute

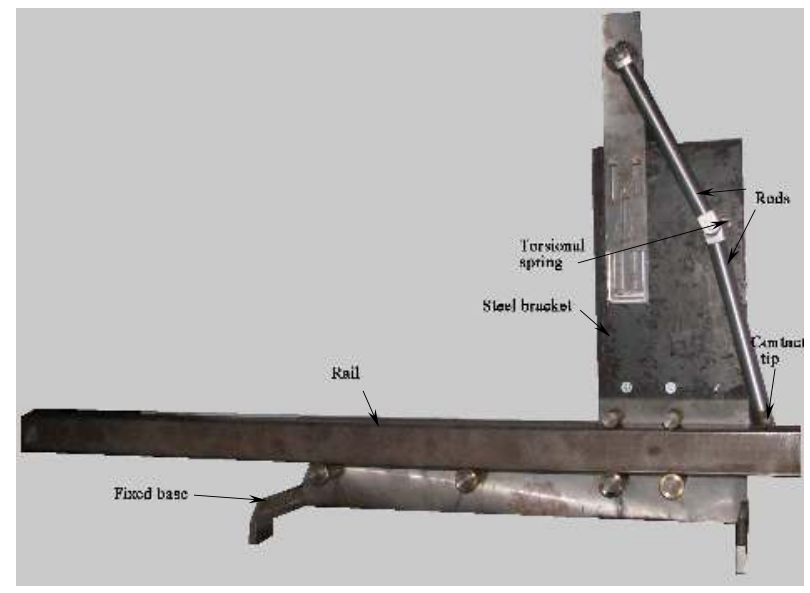

Figure 2: The physical model of the experimental setup

joint. To consider the effects of joint friction, we use a uniform coefficient $c$ to represent the damping torques acting on the two identical revolute joints. So the torques $\tau_{1}$ and $\tau_{2}$ at the revolute joints can be approximated as

$$
\begin{aligned}
& \tau_{1}=-c \dot{\theta_{1}} \\
& \tau_{2}=k\left(\theta_{1}-\theta_{2}-\alpha_{0}\right)+c\left(\dot{\theta_{1}}-\dot{\theta_{2}}\right)
\end{aligned}
$$

where $k$ is the stiffness of the torsional spring, and $\alpha_{0}$ is the initial angle of the spring.

Two laser-Doppler vibrometers (OFV-303-353) with a controller(OFV-3001) are used to measure the rail speed and the velocity of the contact point, in which laser signals are sent to track the movements of the sensitive papers attached in the rail and the contact head. The experimental signals are transferred into a laptop through an $A \backslash D$ card with $10 \mathrm{kHz}$ sample rate. The sketch of the experimental system is depicted in figure 3 .

By using a simple slide experiment for the homogenous contact surface, we estimate the coefficient of friction as $\mu=0.6$. Meanwhile, the contact constraint will allow the following geometric relationship to exist

$$
l\left(\cos \theta_{1}+\cos \theta_{2}\right)=H
$$

where $H$ is the height of the system (see figure 1), and $\theta_{1}$ and $\theta_{2}$ are the angles related to the upper joint and the middle joint, respectively. 


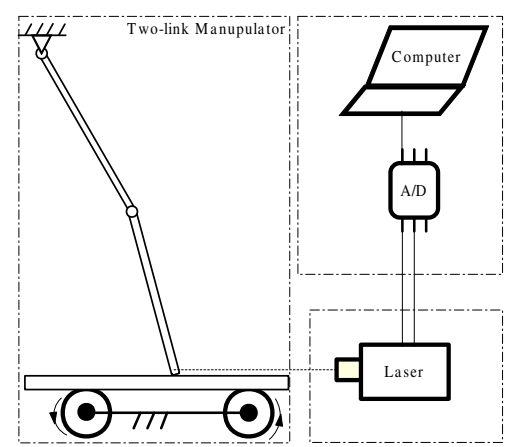

Figure 3: The sketch of the experimental system

Based on the above relationship and one the condition $A<0$, a function of $\theta_{1}$ with respect to $H$ and $\mu$ can be obtained for the occurrence of the Painlevé paradox. For the given coefficient of friction $\mu=0.6$ with different value of $H$, Table 1 presents the allowable scope of $\theta_{1}$ for the occurrence of the Painlevé paradox.

Table 1: The paradoxical configurations in different height

\begin{tabular}{|c|c|c|c|c|}
\hline & \multicolumn{4}{|c|}{ The coefficient of friction $\mu=0.6$} \\
\hline$\theta_{1}(\mathrm{deg})$ & none & $41.5 \sim 48.5$ & $33 \sim 42.5$ & $22.7 \sim 34.9$ \\
\hline$H(\mathrm{~m})$ & 0.21 & 0.331 & 0.35 & 0.3775 \\
\hline
\end{tabular}

Since the paradoxical situations appear under the condition that the robotic system should be in a paradoxical configuration with an initial condition of slip, we can release the tip of the system with an approximately zero height on the moving rail in order to generate the slip mode. In this case, the absolute value of the velocity of the tip is equal to zero, such that a relative slip between the tip and the rail can be established. According to the property of the tangential impact, the absolute tangential velocity of the tip should immediately approach the one of the moving rail if a shock exists. In the following, we will present the experimental results for the robotic system with different configurations by changing the joint angles and the height of the system. In particular, the observation of the stick phenomena associated with the paradoxical situations will be emphasized.

\subsection{The experimental results}

Table 2: The configurations investigated in experiments

\begin{tabular}{|c|c|c|c|c|c|c|c|}
\hline & \multicolumn{6}{|c|}{ The height $H=0.3775 m$} \\
\hline$\theta_{1}($ deg $)$ & -15 & 7 & 15 & 21 & 25 & 30.5 & 32 \\
\hline Paradox & $\mathrm{N}$ & $\mathrm{N}$ & $\mathrm{N}$ & $\mathrm{N}$ & $\mathrm{Y}$ & $\mathrm{Y}$ & $\mathrm{Y}$ \\
\hline
\end{tabular}


Let us set the robotic system with a fixed height $H=0.3775 \mathrm{~m}$, then repeat experiments for the system with joint angle $\theta_{1}$ that takes different values among the scope of $\theta_{1} \in\left(-37^{\circ}, 37^{\circ}\right)$ (the possible values that can make the tip of the robotic system touch on the moving rail). The negative value of $\theta_{1}$ corresponds to the situation where rod 1 slopes to the left side against the vertical line passing through the fixed revolute joint. Table 2 presents the configurations of the system that are investigated in experiments, in which "Y" represents the paradoxical configurations, while "N" represents the ones of non paradox appearing.

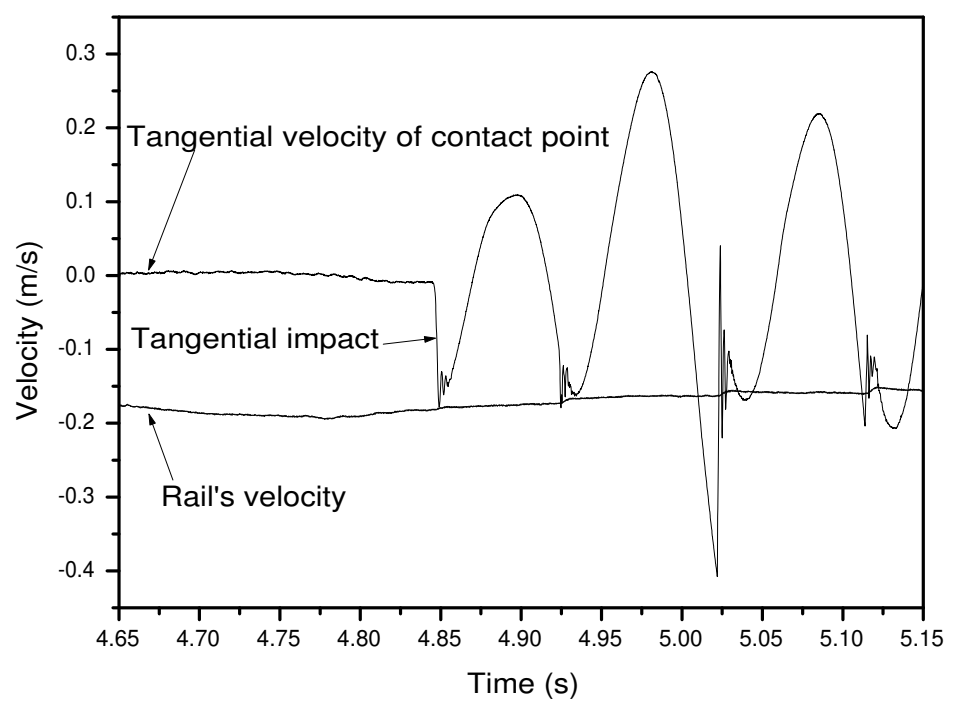

Figure 4: The relative velocity of the contact point in tangential direction $(H=0.3775 \mathrm{~m}$, $\left.\theta_{1}=32^{\circ}, v_{t}=-0.16 \mathrm{~m} / \mathrm{s}\right)$

Figure 4 shows the experimental curves for the rail's speed and the tangential velocity of the contact point for the system with a paradoxical configuration of $\theta_{1}=32^{\circ}$. When the tip touches the moving rail with zero velocity at $t=4.84 \mathrm{~s}$, the first vertical line shown in figure 4 indicates that the tip immediately approaches the value of the rail speed. This is related to a sticking phenomenon corresponding to a tangential impact appearing in the paradoxical configuration. After this event the tip bounces on the moving rail and sequential collisions appear at $t=4.92 \mathrm{~s}, 5.02 \mathrm{~s}, 5.10 \mathrm{~s}$, etc.

We also found from experiments that the magnitude of the bouncing motion will be significantly influenced by the rail's speed. Figure 5 presents the experimental results by setting the robotic system in the same configuration as in the previous experiments, while the rail's speed is changed to $v_{t}=-0.5 \mathrm{~m} / \mathrm{s}$. A tangential impact appears at the measure time $t=2.12 \mathrm{~s}$ when the tip touches the rail, and then the subsequent impacts occur at the instant $t=2.21,2.29, \ldots$. The comparison between figures 4 and 5 clearly shows that the magnitude of the tangential velocity is enlarged due to the increase of the rail's speed.

If the rail moves slowly, the bouncing motion induced by the tangential impact will be of low magnitude and even disappear when the velocity of the rail is lower than a 


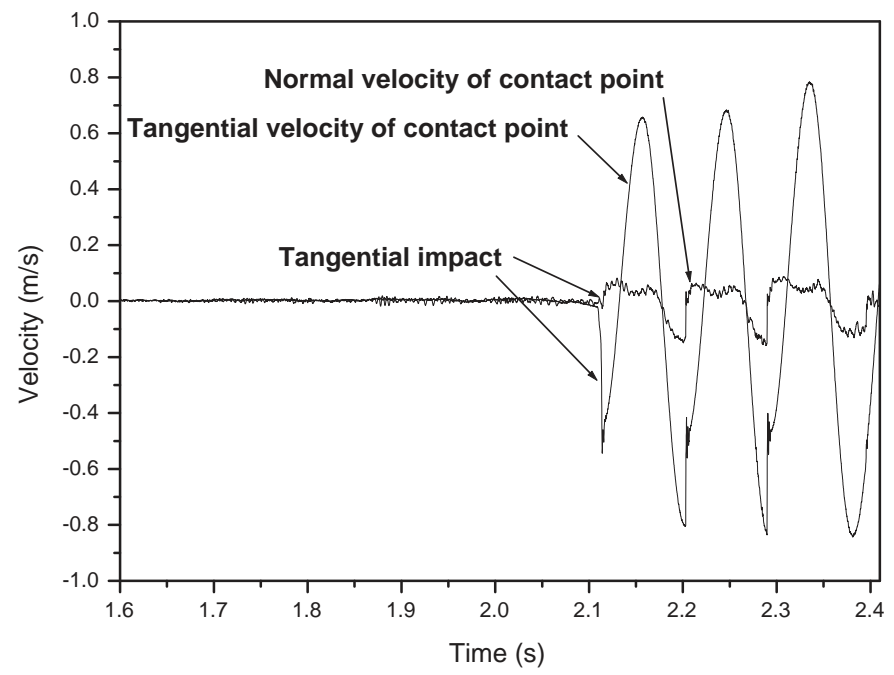

Figure 5: The normal and tangential velocity of the contact point $\left(H=0.3775 m, \theta_{1}=\right.$ $\left.32^{\circ}, v_{t}=-0.5 \mathrm{~m} / \mathrm{s}\right)$

certain threshold. Figure 6 shows the experimental results for the robotic system with the same configurations as the previous two experiments, while setting the rail moving with $v_{t}=-0.075 \mathrm{~m} / \mathrm{s}$. Clearly after the tangential impact the tip of the robotic system will stick on the moving rail, even though there is a peak in the curve of the tangential velocity because of the tangential impact.

This phenomenon can be elucidated from the viewpoint of the system's energy. According to equations (18) and (19), the normal and tangential impulses are proportional to the rail's speed. So the robotic system can gain more energy from the tangential impact in the situation of the rail moving fast. Thus the sequential collisions can be enlarged to make the magnitude of the bouncing motion increase. If the rail moves much more slowly, the robotic system cannot gain enough energy to overcome the contact force generated by the gravity and the torsional spring. In this case, the bouncing motion cannot be observed experimentally.

For the system with the configuration of $\theta_{1}=30.5^{\circ}$, the bouncing motion due to the tangential impact can also be observed by experiments when the rail takes a relatively high speed (as shown in figure 17). The experimental results related to the two cases of the system with $\theta_{1}=30.5,32^{\circ}$ will be used in the following section to verify the numerical simulations.

When $\theta_{1}<29^{\circ}$, the joint angle $\theta_{2}$ will be greater than $\theta_{1}$ if the tip can touch on the rail for the system with height $H=0.3775 \mathrm{~m}$, so that the initial torque of the torsional spring applied at the middle joint will change its direction and then influences the dynamical behavior of the system. Figure 7 presents the experimental results for the system with a paradoxical configuration by setting joint angle $\theta_{1}=25^{\circ}$. When contact is established, a tangential impact appears at the contact point (the tangential velocity of the tip immediately approaches the one of the rail), and then an oscillation for the tangential motion 


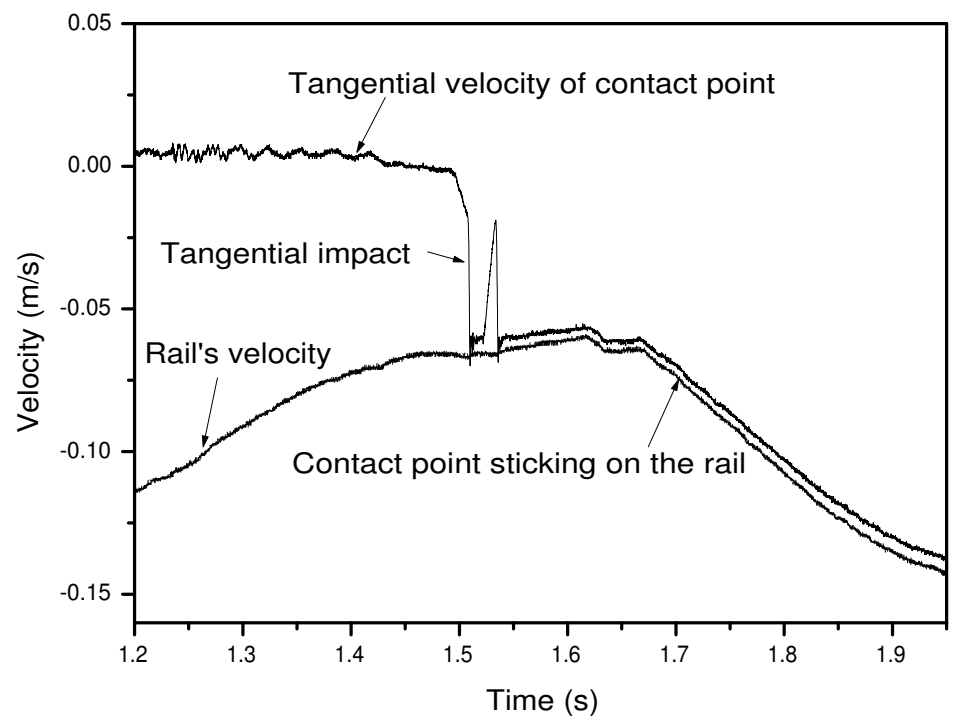

Figure 6: The tangential velocity of the contact point $\left(H=0.3775 m, \theta_{1}=32^{\circ}, v_{t}=\right.$ $-0.075 \mathrm{~m} / \mathrm{s}$ )

is induced. However, the contact point doesn't leave away from the rail surface after the tangential impact and no bouncing motion can be observed. The reason for that is because the system with the configurations of $\theta_{2}>\theta_{1}$ can only obtain very little energy from the tangential impact that is not enough to overcome the effects of the gravity and the torsional spring. Therefore, the contact point will stick on the moving rail.

Although the above experimental investigations indicate that the dynamical behavior of the system with paradoxical situations seems to be well modeled as a tangential impact as the relative tangential velocity could immediately approach zero, it is clear in physical meaning that the tangential motion with a normal configuration evolves into a shock for the paradoxical configurations should correspond to a process with gradual change in tangential velocity. Figure 8 to 11 present the experimental results obtained by setting the system with $\theta_{1}=-15,7,15,21^{\circ}$, respectively. This corresponds to the situation where the initial configuration of the system gradually approaches the boundary of the singular region. From the experimental curves (except for the case of $\theta_{1}=-15^{\circ}$ ), we can find that friction will decrease the slip velocity and finally bring the contact point to stick on the moving rail. In particular, the duration from the beginning of slip to the occurrence of sticking becomes shorter when the configuration of the system is near to the paradoxical situation. If the configuration is very close to the boundary of the paradoxical region (the case of $\theta_{1}=21^{\circ}$ ), the duration for the stop of the relative tangential motion is about $t=0.13 \mathrm{~s}$. Once the configuration of the system enters into the paradoxical region, the duration for the tangential motion of the contact point changing from slip to stick will be less than $0.01 \mathrm{~s}$ (as shown for the case of $\theta_{1}=25^{\circ}$ ), a short timescale that can be connected with an impact process. The above experimental phenomena well agree with the mechanism of the Painlevé paradox generated due to the coupling between friction and the configuration of the system, and further confirm that the dynamical behavior of 


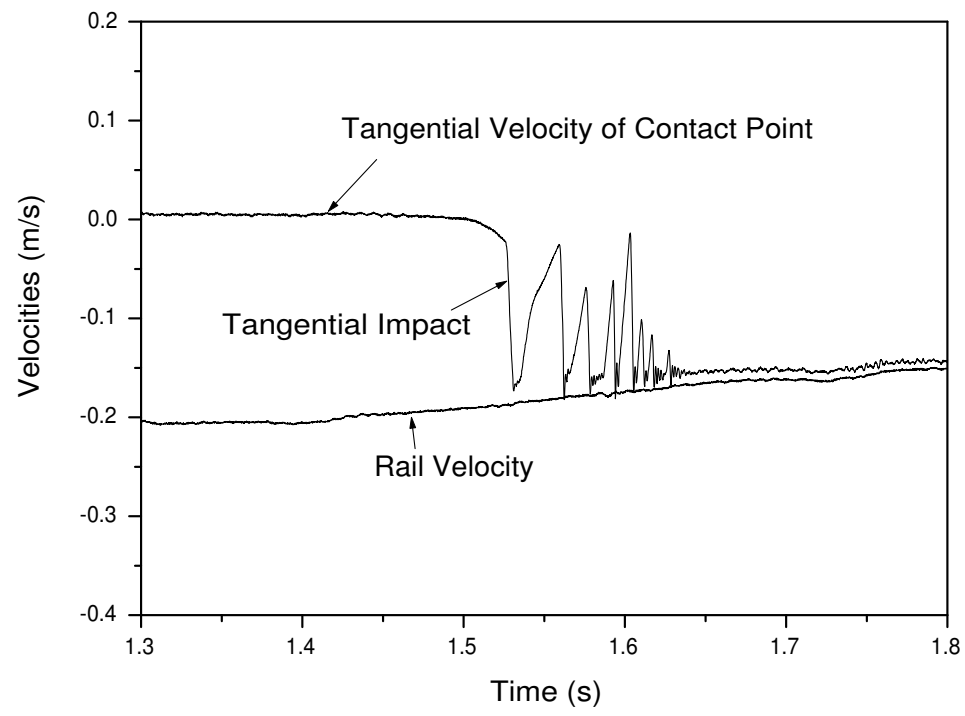

Figure 7: The tangential velocity of the tip with $H=0.3775 \mathrm{~m}$ and $\theta_{1}=25^{\circ}$

the system in the Painlevé paradox could be described by a shock.

If the initial configuration is far away the singular region, as shown in figure 8 for the system with a configuration of taking $\theta_{1}$ with a negative value and in figure 12 for the robotic system with the initial configuration of $H=0.25 \mathrm{~m}$, neither sticking phenomena nor the bouncing motion can be found in the robotic system.

In some cases, the stick-slip phenomenon is also found in our experiments for the robotic system. By setting the initial configuration of the system to $H=0.314 m$ and $\theta_{1}=50^{\circ}$, figure 13 shows that the friction will bring the tip into a stick, and then make the slip resume. Such a process can be repeated to render the robotic system unstable on the contact surface.

\section{The comparison between experimental and numerical re- sults}

The experimental results presented in the above section have confirmed that the Painlevé paradox does induce a tangential impact at the contact point, and then makes the robotic system behave in a more complex way, with slip phases, stick phases, flight without contact phases, as well as tangential and normal impacts with friction. In this section, we will use an event-driven algorithm to perform the numerical simulations.

Firstly, we will carefully estimate the parameters used for the simulations. According to property of the collision between plastic and steel materials, the coefficient of the restitution can be set as $e_{p}=0.1$. A single pendulum system (shown in figure 14 is established to obtain its frequency $f$, and the value of the stiffness of the torsional spring 


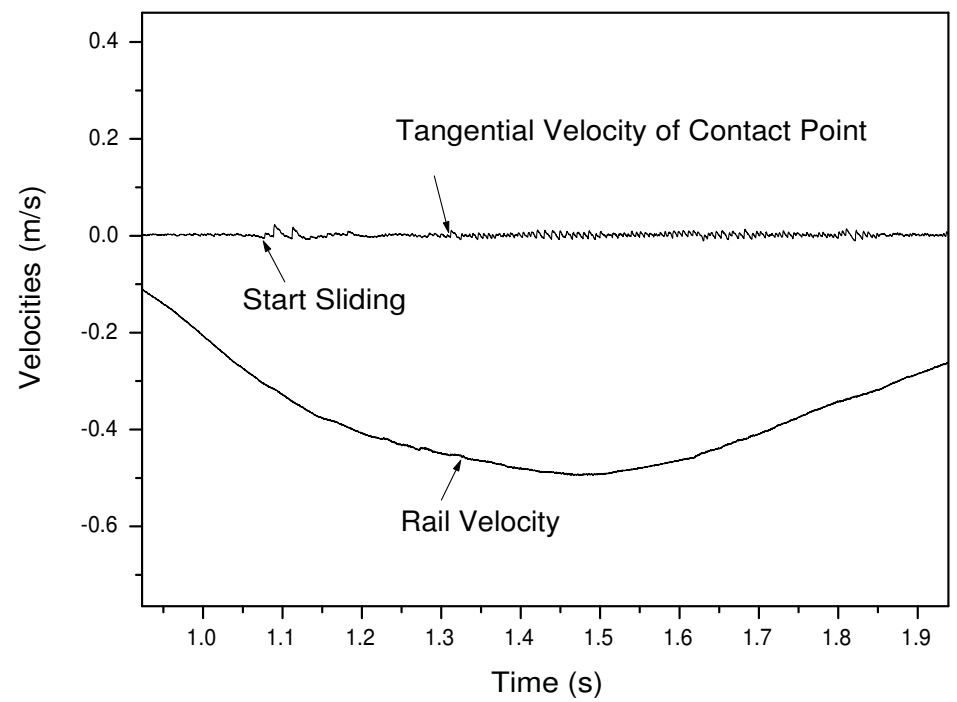

Figure 8: The tangential velocity of the tip with $H=0.3775 m$ and $\theta_{1}=-15^{\circ}$

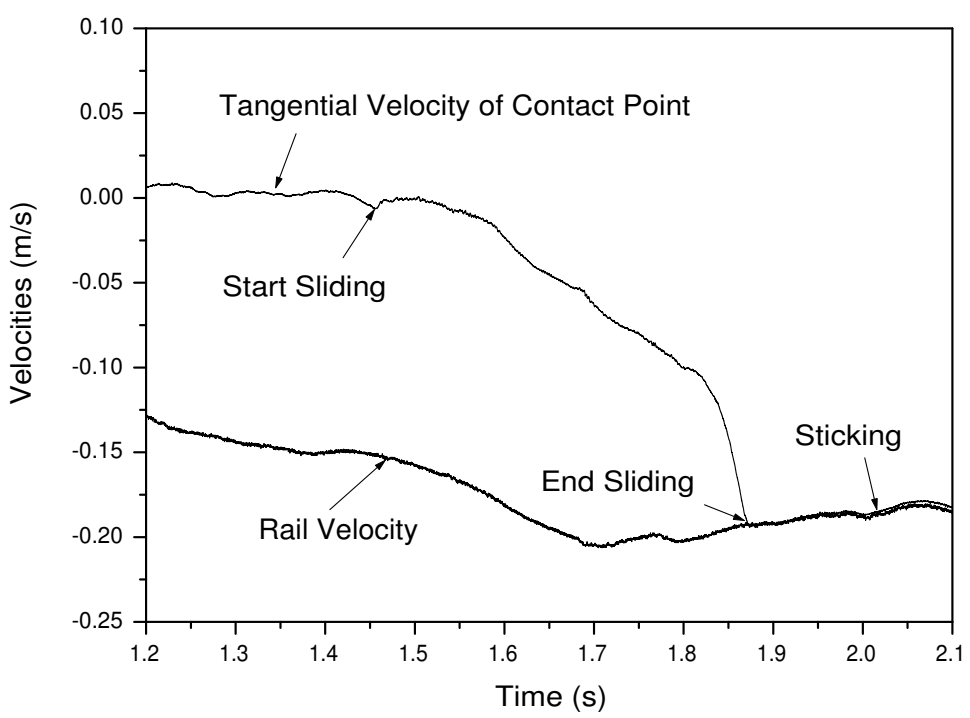

Figure 9: The tangential velocity of the tip with $H=0.3775 m$ and $\theta_{1}=7^{\circ}$ 


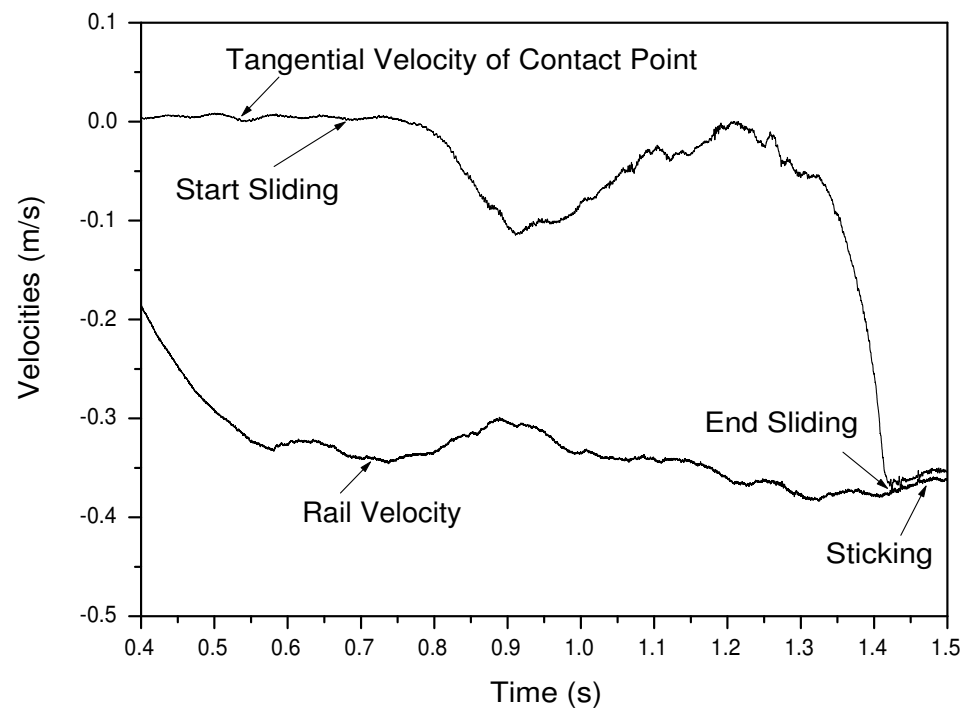

Figure 10: The tangential velocity of the tip with $H=0.3775 \mathrm{~m}$ and $\theta_{1}=15^{\circ}$

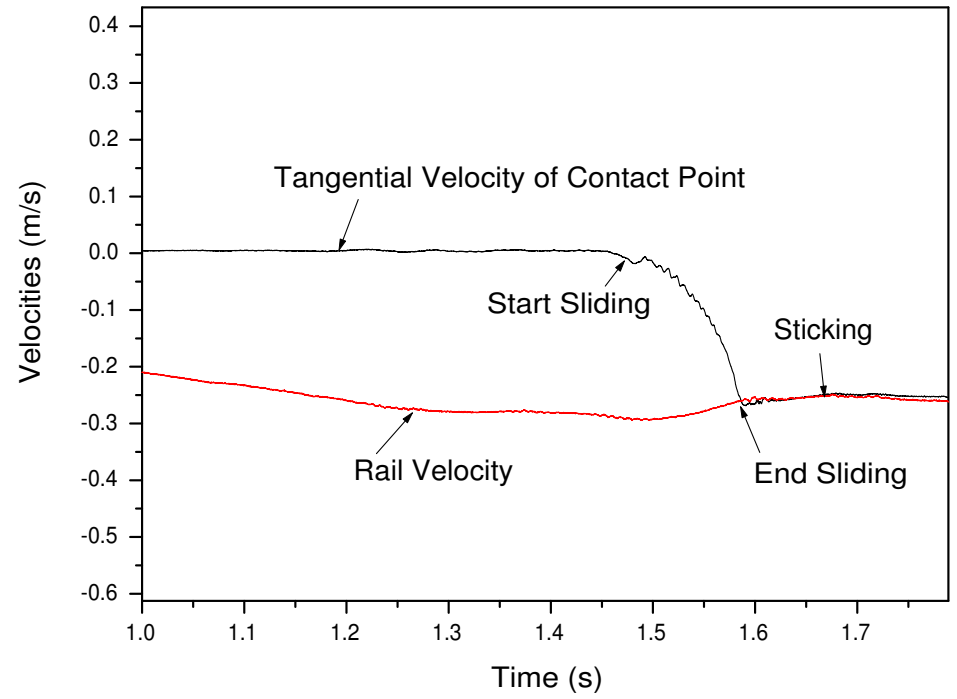

Figure 11: The tangential velocity of the tip with $H=0.3775 \mathrm{~m}$ and $\theta_{1}=21^{\circ}$ 


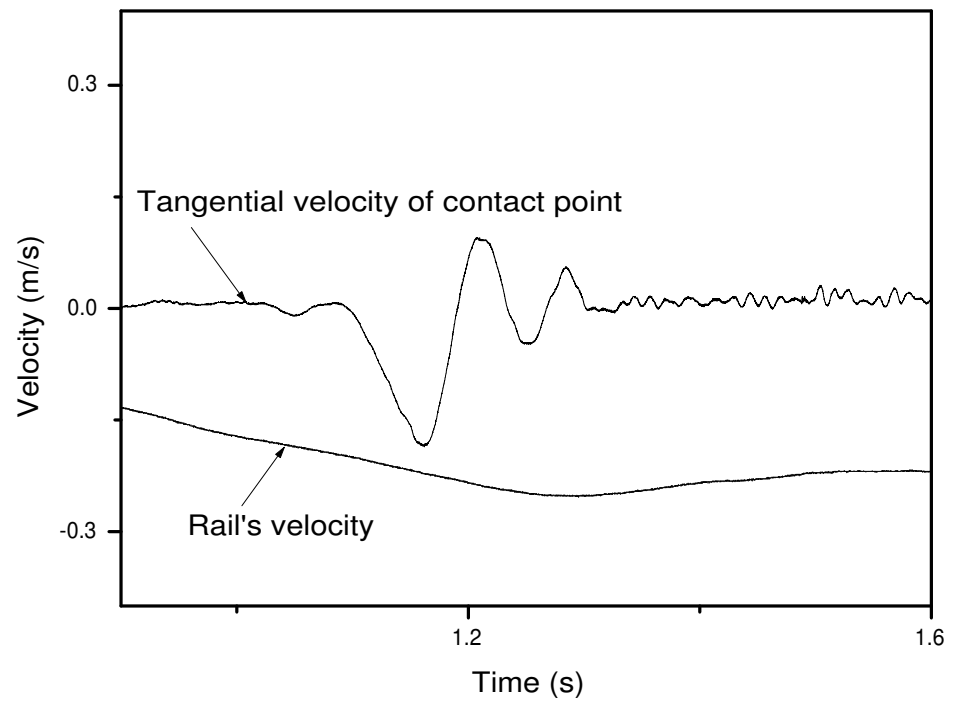

Figure 12: The tangential velocity of the tip without the Painlevé paradox $(H=0.25 \mathrm{~m}$, $\left.\theta_{1}=69.4^{\circ}\right)$

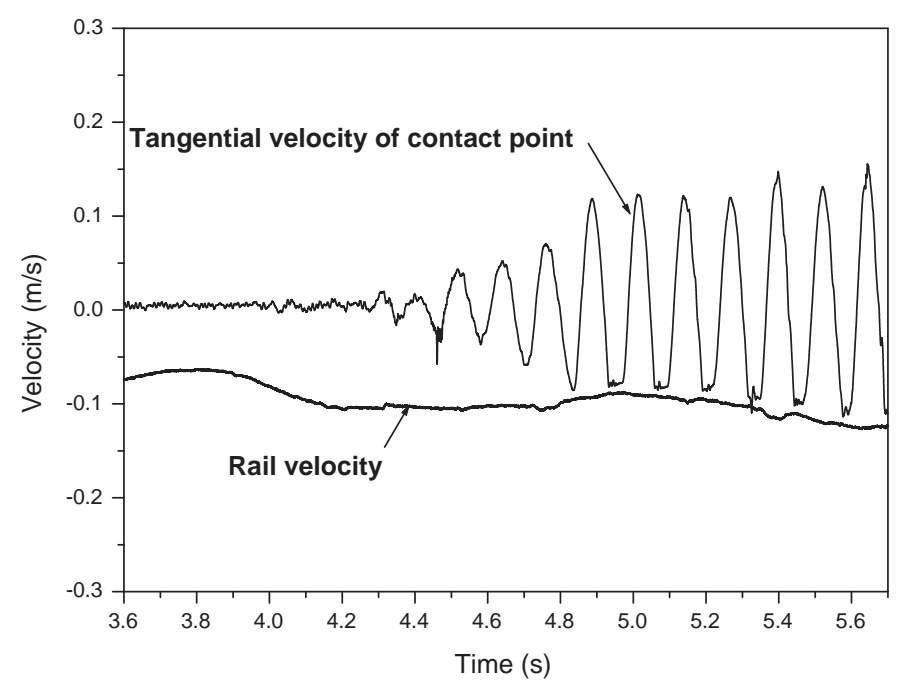

Figure 13: The stick-slip phenomenon in the configuration $\left(H=0.314 m, \theta_{1}=50^{\circ}\right)$ 


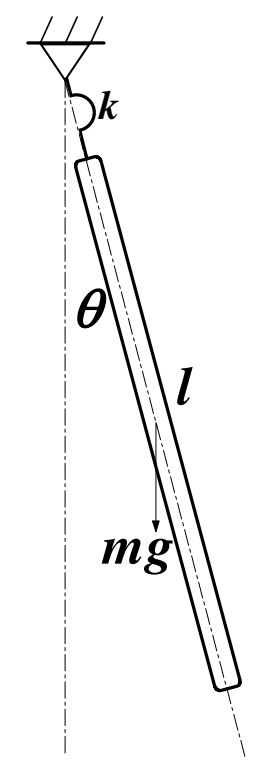

Figure 14: A single pendulum composed by a link and a torsional spring

$k$ can be calculated by using the following expression:

$$
k=\frac{1}{3} m l^{2}(2 \pi f)^{2}-\frac{l}{2} m g
$$

Based on $f=4.6 \mathrm{~Hz}$ measured from experiments, we have $k=1.3 \mathrm{~N} \cdot \mathrm{m} / \mathrm{rad}$.

Since the damping coefficient $c$ is difficult to obtain from the experiments, a fitting method is used for its estimation. By setting $c$ with different values to perform the simulations, we choose the best one among the different values of $c$ as the damping coefficient that can make the corresponding numerical simulation better coincide with the experimental results. According to the numerical experiments, we find that the change of $c$ limited in the range of $[0.003,0.007]$, has little influence on the numerical results. So the damping coefficient is chosen as $c=0.005 \mathrm{Nms} / \mathrm{rad}$ for the following simulations.

Roughly speaking, the dynamical behaviors of the system are governed by equations (4) and (5) with the variations of the contact forces, which depend on the mode of motion at the contact point. For instance, the contact forces can be set to zero for the flying mode, while in the case of preserved contact, the contact forces should be determined by using LCP's equations and the Coulomb's friction law. If the paradoxical situations appear in the rigid body model, the simulation can be continued by setting equation (4) with new initial conditions that can be obtained from the tangential impact rule expressed in (18) and (19). Similar process is also carried out for the collisions with friction [21], in which the changes of the velocities of the system are obtained by integrating the impulsive differential equations expressed in (12). Since there are no accumulations of events, event-driven schemes are well-suited to the numerical integration of this nonsmooth system, see e.g. [40].

Figure 15 presents the comparisons between the experimental and numerical results by setting the system to the initial configuration of $H=0.3775 \mathrm{~m}$ and $\theta_{1}=32^{\circ}$. It is 
clear that the model used in simulation can well reproduce the qualitative behaviors of the system. The discrepancies appearing in figure 10 are partly due to the unmodeled effects existing in the experimental setup, such as the vibration of the sensitive papers, the clearances in the revolute joints, and the errors of the physical parameters. Other sources of discrepancies are the non-uniformity of the rail's speed in experiments, since it is assumed to be a constant in the numerical simulations.

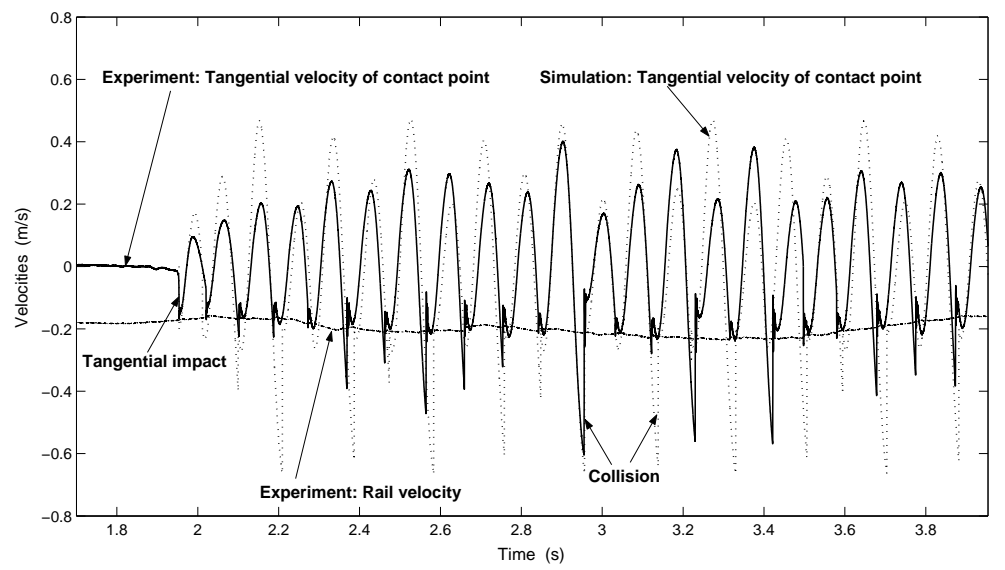

Figure 15: Experimental and numerical results for the tangential speed $(H=0.3775 m$, $\left.\theta_{1}=32^{\circ}, v_{t}=-0.2 \mathrm{~m} / \mathrm{s}\right)$

Figure 16 shows the comparisons of the normal velocities between the experimental and numerical results. The vibration of the sensitive paper induced by the bouncing motion will much influence the accuracy of the measurements. Nevertheless, the qualitative behavior of the system can still be captured through the numerical simulations.

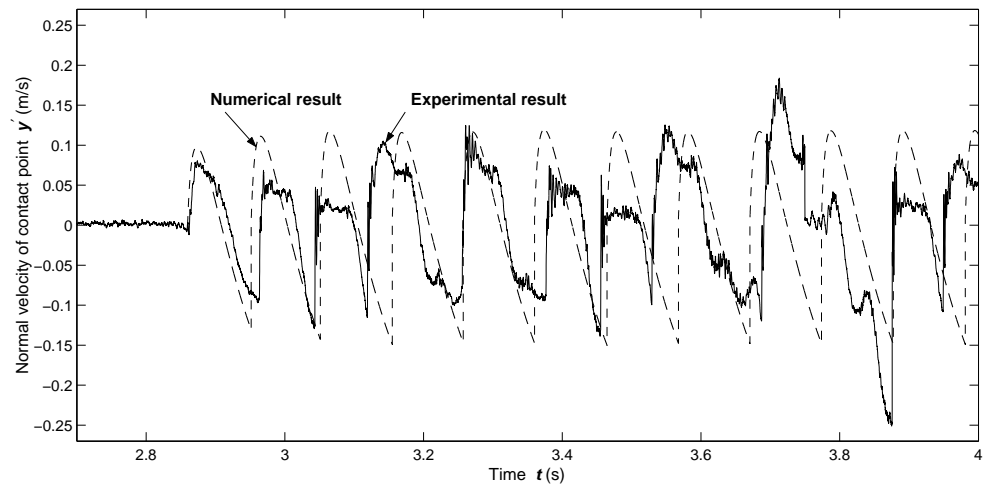

Figure 16: Experimental and numerical results for the normal velocity $(H=0.3775 m$, $\left.\theta_{1}=32^{\circ}, v_{t}=-0.24 \mathrm{~m} / \mathrm{s}\right)$

Keeping the system with the same height $H=0.3775 \mathrm{~m}$ as in the previous case, we can change the initial configuration by adjusting the joint angle from $\theta_{1}=32^{\circ}$ to $30.5^{\circ}$. The results obtained from the simulation also agree well with the experimental results (shown in figure 17).

As mentioned in the above subsection, the phenomenon of stick-slip motion can be 


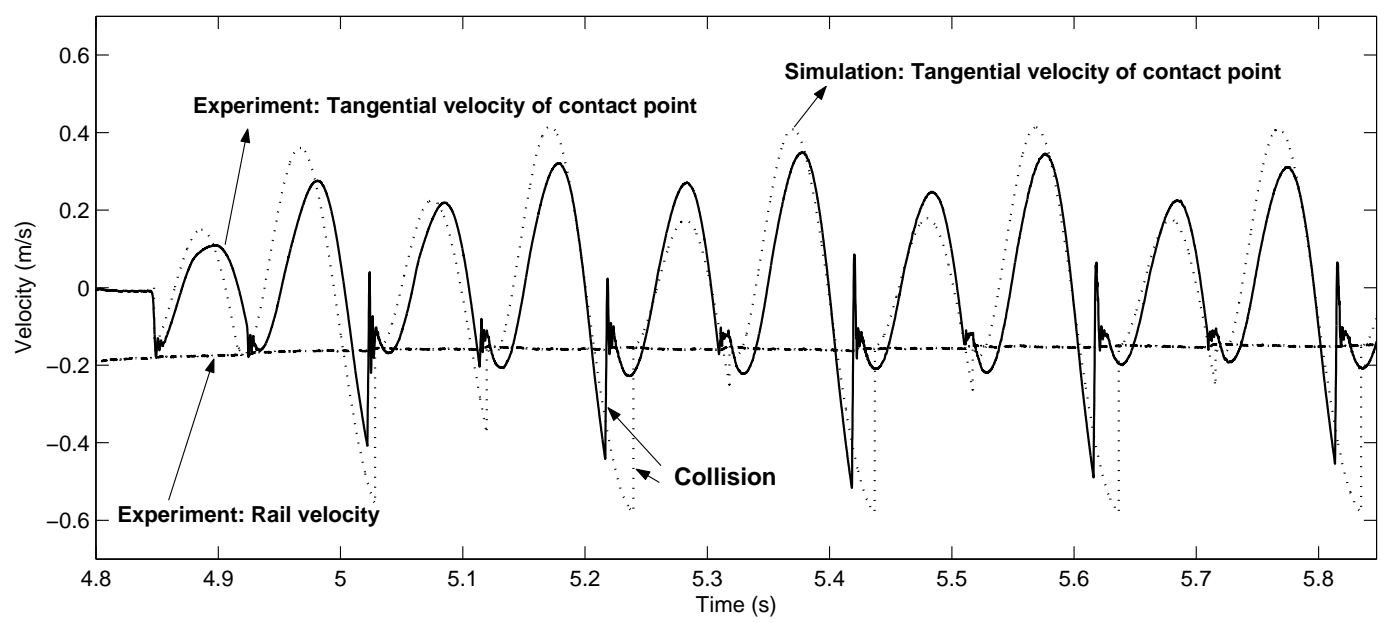

Figure 17: The comparison of the tangential speed between experimental and numerical $\operatorname{results}\left(H=0.3775 m, \theta_{1}=30.5^{\circ}, v_{t}=-0.2 \mathrm{~m} / \mathrm{s}\right)$

found in experiments even though there is no paradox appearing. By setting the initial configuration of the system with $H=0.314 m$ and $\theta_{1}=55^{\circ}$, figure 18 presents the curves corresponding to the tangential velocities of the contact point obtained from simulations and experiments, respectively. The numerical results indicate that the stick-slip is a pure periodic motion when the rail speed takes a constant value, but this characteristic will be slightly destroyed due to the non-uniformity of the rail's motion.

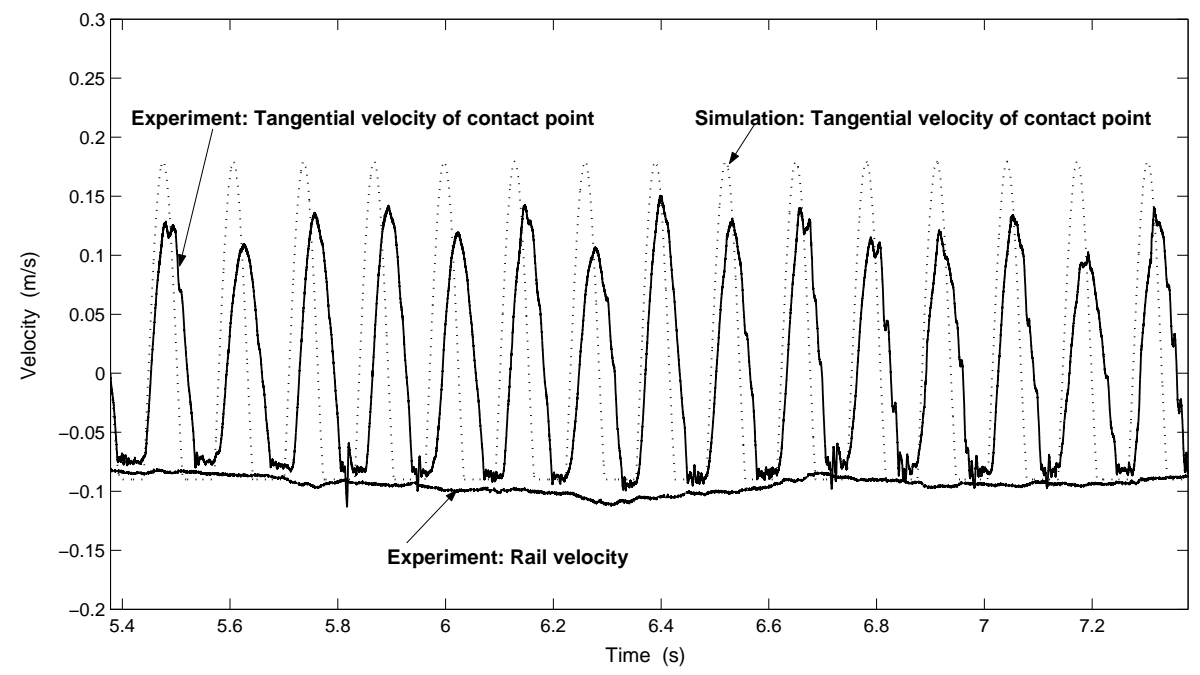

Figure 18: Comparison between the experimental and numerical results for the Stick-Slip Phenomena under the configuration taken as $H=0.314 \mathrm{~m}$ and $\theta_{1}=55^{\circ}$

Summarizing the comparisons between the numerical and experimental results presented in above, we can conclude that the dynamical behaviors of the system can be well captured qualitatively by using the rigid body model. Even in the paradoxical situation, 
the simulations can be led by using the tangential impact rule to reinitialize the dynamical equations. The numerical results show that such a tangential impact induced by the Painlevé paradox can be well governed by the impact rule presented in [28].

\section{Summary and conclusion}

This study mainly developed an experimental setup to demonstrate the phenomenon of the Painlevé paradox that appears in a robotic system coming into contact with a moving rail. According to the experimental results, we have verified that a shock is truly related to the problem of the Painlevé paradox, and takes a particular property that a tangential stick appears at the contact point.

Two kinds of instability phenomena for the robotic system are observed from the experiments. The first one is induced by the tangential impact, in which the robotic system will bounce or stick on the moving rail, depending on the value of the rail's speed. The other form of the instability is the stick-slip motion, which appears in the system does not involve the Painlevé paradox.

Based on the careful estimation of the physical parameters, an event-driven algorithm is used to perform numerical simulations. By setting the robotic system with paradoxical situations, comparisons between the numerical and experimental results are carried out and show good agreement. This illustrates that the rigid body model can well reproduce the complexly dynamical behaviors, and the Painlevé paradox can be overcome by using the tangential impact rule obtained from the Darboux-Keller's shock dynamics. The present work not only provides a basis for the theoretical results associated with the problem of the Painlevé paradox, but also may be useful for the design of feedback controllers in robotic systems.

\section{Acknowledgements}

We would like to express our thanks to Dr Bernard Brogliato for his valuable comments and the suggestions to this paper. We also appreciate the assistance from Dr. Zhili Sun, Jiyan Huo and Ming Qiang to the work of experiment. The research was supported by Chinese National Science Foundation (Grant No. 60334030, 10642001, 10772002).

\section{References}

[1] Y. Hurmuzlu, F. Génot, B. Brogliato, Modeling, stability and control of biped robots - a general framework. Automatica, 40: 1647-1664, 2004

[2] F. Pfeiffer and C. Glocker, Multibody Dynamics with Unilateral Contacts, Wiley, New York, 1996

[3] W.J. Stronge, Impact Mechanics, Cambridge University Press, 2000

[4] B. Brogliato, Some Perspectives on the Analysis and Control of Complementarity Systems, IEEE Trans. on Automatic Control, 48(6), 918-935, 2003 
[5] B. Brogliato. Nonsmooth Mechanics, 2nd edition. Springer, London, 1999

[6] P. Painlevé, Sur les lois du frottement de glissement, Comptes Rendu des Séances de l'Academie des Sciences, 121, 112-115, 1895

[7] F. Klein, Zu Painlevés Kritik der Coulombschen Reibungsgesetze. Zeit. Math. Physik, 58: 186-191, 1909

[8] P. Lötstedt, Coulomb friction in two-dimensional rigid-body systems. Z. Angew. Math. Mech., 61: 605-615, 1981

[9] P. Lötstedt, Mechanical systems of rigid bodies subject to unilateral constraints. SIAM J. Appl. Math., 42: 281.296, 1982

[10] M. Erdmann, On a representation of friction in conguration space. Int. J. Robotics Research, 13(3):240-271, 1994

[11] J. J. Moreau, Unilateral contact and dry friction in finite freedom dynamics. Nonsmooth Mechanics and Applications, pp. 1-82. Springer.Verlag, Vienna, New York, 1988

[12] Y. Wang and M. T. Mason, Two-dimensional rigid-body collisions with friction. J. Appl. Mech., 59: 635.642, 1992

[13] D. Baraff, Coping with Friction for Non-penetrating Rigid Body Simulation, Computer Graphics, Vol. 25, No. 4, 31-40, 1991

[14] M. Payr and C. Glocker, Oblique Frictional Impact of a Bar: Analysis and Comparison of Different Impact Laws, Nonlinear Dynamics 41: 361-383, 2005

[15] R.I. Leine, B. Brogliato and H. Nijmeijer, Periodic motion and bifurcations induced by the Painlevé paradox; European Journal of Mechanics A/Solids 21: 869-896, 2002

[16] F. Génot and B. Brogliato, New results on Painlevé paradoxes. European Journal of Mechanics A/Solids, 18: 653-677, 1999

[17] A.P. Ivanov, The problem of constrainted Impact, J. Appl. Maths Mechs, Vol.61 No.3:341-253, 1997

[18] A.P. Ivanov, Singularities in the dynamics of systems with non-ideal constraints. J. Appl. Maths. Mechs, Vol. 67, No. 2, pp. 185-192, 2003

[19] R.M. Brach, Impact Coefficients and Tangential Impacts. Trans. ASME. J. of App. Mech., 64: 1014-1016, 1997

[20] Z. Zhao, B. Chen, C. Liu, Impact Model Resolution On Painlevé's Paradox. ACTA Mechanica Sinica, 20(6):659-660, 2004

[21] Z. Zhao, C.Liu and B.Chen, The Numerical Method for Three-Dimensional Impact with Friction of Multi-Rigid-Body System. Science in China Series G-Physics and Astronomy. Vol.49(1): 102-118, 2006 
[22] S. Peng, P. Kraus, V. Kumar and P. Dupont, Analysis of rigid-body dynamic models for simulation of systems with frictional contacts. Journal of Applied Mechanics,68: $118-128,2001$

[23] S.S. Grigoryan, The Solution to the Painlevé Paradox for Dry Friction, Doklady Physics, Vol. 46, No. 7: 499-503,2001

[24] D.E. Stewart, Rigid-Body Dynamics with Friction and Impact SIAM Review, Vol. 42(1): 3-39, 2000

[25] D.E. Stewart, Convergence of a Time-Stepping Scheme for Rigid-Body Dynamics and Resolution of Painleve's Problem; Arch. Rational Mech. Anal. 145: 215-260, 1998

[26] E.V. Wilms and H. Cohen, The occurrence of Painlevé's paradox in the motion of a rotating shaft. Journal of Applied Mechanics 64, pp.1008-1010, 1997

[27] R.A. Ibrahim, Friction-induced vibration, chatter, squeal and chaos. Part ii: Dynamics and modeling. ASME Applied Mechanics Reviews 47 (7): 227-253,1994

[28] C.Liu, Z.Zhao, B. Chen, The Bouncing Motion Appearing in a Robotic System with Unilateral Constraint, Nonlinear Dynamics, 49 (1-2): 217-232, 2007

[29] Z.Zhao, C.Liu, B. Chen, The Painlevé Paradox in a 3D Slender Rod, (accepted).

[30] M.Anitescu, F.A. Potra, Time-stepping schemes for stiff multi-rigid-body dynamics with contact and friction. Int. J. Numer. Methods Eng. 55(7), 753C784, 2002

[31] M. Anitescu, F.A. Potra, D. Stewart: Time-stepping for three-dimensional rigidbody dynamics. Comput. Methods Appl. Mech. Eng. 177, 183C197, 1999

[32] T.R. Kane T R, D.A. Levinson, Dynamics: Theory and Applications. New York: McGraw- Hill Ltd, 1985.

[33] W.J. Stronge, Swerve during three-dimensional impact of rough rigid bodies. Transactions of ASME Journal of Applied Mechanics, 1994, 61: 605611

[34] J.B. Keller, Impact with friction. Transactions of ASME Journal of Applied Mechanics, 53: 1-4, 1986

[35] V. Bhatt and J.Koechling, Partitioning the parameter space according to different behaviors during three-dimensional impacts. Transactions of ASME Journal of Applied Mechanics, 62: 740-746,1995

[36] J.A. Batlle, Rough balanced collisions. Transactions of ASME Journal of Applied Mechanics, 63: 168-172,1996

[37] G. Darboux, Etude Géométrique sur les percussions et le choc des corps, Bulletin des Sciences Mathématiques et Astronomiques, deuxième série, tome 4, 126-160, 1880

[38] J.J. Moreau, Les liaisons unilatérales rt le principe de Gauss, C.R. Acad. Sciences Paris, 256, 871-874, 1963 
[39] J.J. Moreau, Mécanique Classique, tome II, Masson, Paris, 1971

[40] B. Brogliato, A.A. ten Dam, L. Paoli, F. Génot, M. Abadie, Numerical simulation of finite dimensional multibody nonsmooth dynamical systems. ASME Applied Mechanics Reviews, vol.55, no 2, pp.107-150, March 2002. 